\title{
EARLY ONSET WEST SYNDROME WITH HYPOMYELINATION, COLOBOMA AND SPTAN1 MUTATION
}

Investigators from Ljubljana, Slovenia report an 8-month-old female infant with hypotonia, lack of visual attention, early onset epileptic encephalopathy, and severe developmental delay. She presented with myoclonic jerks at 6 weeks and infantile spasms at age 3.5 months, accompanied by a hypsarrhythmia pattern EEG. Seizures were resistant to pyridoxine, levetiracetam, vigabatrin, and hydrocortisone. Topiramate was of some benefit, but the EEG progressed to a suppression-burst-like pattern. Brain MRI at 10 weeks of age revealed hypomyelination in the internal capsule and central regions, thinning of corpus callosum, and cortical atrophy. Eye exam showed dysplastic, coloboma-like optic discs. A heterozygous deletion was detected in SPTAN1 gene. (Writzl K, Primec ZR, Strazisar BG, et al. Early onset West syndrome with severe hypomyelination and coloboma-like optic discs in a girl with SPTAN1 mutation. Epilepsia 2012 June;53(6):e106-e110). (Respond: Karin Writzl MD, PhD, Institute of Medical Genetics, University Medical Centre, 1000 Ljubljana, Slovenia. E-mail: karinwritzl@gmail.com).

COMMENT. Mutations in the alpha-II-spectrin (SPTANI) gene are previously described in 2 Japanese children with early onset West syndrome, hypomyelination, and hypotonia. (Saitsu et al, 2010, cited in Slovenia article). The Slovenia case expands the phenotypic spectrum to a Caucasian child and with coloboma-like optic discs. All 3 patients had deceleration of rate of head growth, resulting in microcephaly. Patients with infantile spasms show mutations to several genes, and the present report supports the theory of a symptomatic basis for the majority of West syndrome cases. (Paciorkowski AR et al. Genetic and biological classification of infantile spasms. Pediatr Neurol 2011 Dec;45:355-367) (Pediatr Neurol Briefs 2012 Jan;26(1):3-4).

\section{SEIZURE DISORDERS}

\section{SEIZURES AND EPILEPSY IN SOTOS SYNDROME}

Clinicians from the Child Neurology Division, Sapienza University of Rome, and 7 other pediatric neurology centers in Italy report a series of 19 Sotos syndrome (SS) patients with febrile seizures (FS) and/or epilepsy during childhood and a long-term follow-up. Fifteen patients were male and 4 female. Mean age at first evaluation was 5 years 2 months (range 4 months-15 years). Minimum follow-up period was 5 years (range 5-19 years). FS were recorded in 11(58\%) patients and afebrile seizures (AFS) in $15(79 \%)$. FS were only simple in 6/11 (55\%), only complex in $1(9 \%)$, and both simple and complex in $4(36 \%)$. AFS were temporal lobe type in $40 \%$, and were responsive to AED therapy. All patients had the typical facial features of SS (high, broad forehead, down-slanting palpebral fissures, and pointed chin), tall stature and macrocephaly, and learning disability. Neurologic disorders associated with SS are hypotonia, incoordination, delayed language and motor development, behavioral abnormalities, and seizures. (Nicita F, Ruggieri M, Polizzi A, et al. Seizures and epilepsy in Sotos 
syndrome: Analysis of 19 Caucasian patients with long-term follow-up. Epilepsia 2012 June;53(6):e102-e105). (Respond: Alberto Spalice, Child Neurology Division, Department of Pediatrics, Policlinico Umberto L Sapienza University of Rome, Viale Regina Elena 324, 00161, Roma, Italy. E-mail: childneurology.sapienzaroma@live.it).

COMMENT. More than half of children with SS have FS and/or AFS, and more than half of those with FS develop epilepsy, temporal lobe type in $40 \%$. Seizures in SS are usually responsive to AED monotherapy.

\section{WISC-IV IN DETECTION OF EPILEPSY-RELATED COGNITIVE IMPAIRMENTS}

Researchers at Alberta Children's Hospital Research Institute, Calgary, AB, Canada, and Epilepsy Center, New York University, NY analyzed WISC-IV scores from 212 children, 106 with epilepsy (46 girls, 60 boys; mean age 11 years), and 106 controls matched for age, gender, ethnicity, and parental education. Full Scale IQ for the epilepsy group was significantly lower than for controls; $36.8 \%$ had FSIQ $<70$ vs $<1 \%$ controls. In children with epilepsy, Working Memory and Processing Speed Index scores were lower than those for Verbal Comprehension and Perceptual Reasoning $(p<0.01)$. Scores were highest on visual and verbal subtests measuring reasoning skills such as Matrix Reasoning, and lowest on Coding. The Coding subtest identified the most children $(28.3 \%)$ with low scores, and the Similarities subtest identified the fewest (16\%). Later age at onset and shorter epilepsy duration were both correlated with higher WISC-IV FSIQ and index scores. Number of AED trials but not seizure frequency was inversely correlated with FSIQ and index scores. FSIQ, index scores, and subtest scores were not related to left or right-hemisphere seizure foci or to MRI findings. The WISC-IV is useful in detection of cognitive deficits related to childhood epilepsy. (Sherman EMS, Brooks BL, Fay-McClymont TB, MacAllister WS. Detecting epilepsy-related cognitive problems in clinically referred children with epilepsy: Is the WISC-IV a useful tool? Epilepsia 2012 June;53(6):1060-1066). (Respond: Elizabeth MS Sherman PhD, Neurosciences Program, Alberta Children's Hospital, 2888 Shaganappi Trail NW, Calgary, T3B 6A8 AB Canada. E-mail: Elizabeth.sherman@albertahealthservices.ca).

COMMENT. Cognitive impairments in children with epilepsy are secondary to the effects of seizures and AEDs and are sometimes primary, associated with abnormal brain circuitry of epilepsy and predating the onset of seizures. The WISC-IV intelligence test is sensitive to cognitive impairments in children with epilepsy but is not sufficient for measuring some cognitive problems such as memory, sustained attention, executive function, and language deficits often present in children with epilepsy (MacAllister WS, Sherman EMS. Evaluation of children and adolescents with epilepsy. In Barr W, Morrison C (Eds) Handbook of the Neuropsychology of Epilepsy. New York, Springer 2012. In press.) Evaluation of the IQ and other neuropsychological functions is an important aspect of the comprehensive management of childhood epilepsy and an essential component of an Epilepsy Center. 